\title{
NOTES
}

Limnol. Oceanogr., 44(7), 1999, 1781-1787

(C) 1999, by the American Society of Limnology and Oceanography, Inc.

\section{Critical depth and critical turbulence: Two different mechanisms for the development of phytoplankton blooms}

\begin{abstract}
A turbulent diffusion model shows that there are two different mechanisms for the development of phytoplankton blooms. One of these mechanisms works in well-mixed environments and corresponds to the classical critical depth theory. The other mechanism is based on the rate of turbulent mixing. If turbulent mixing is less than a critical turbulence, phytoplankton growth rates exceed the vertical mixing rates, and a bloom develops irrespective of the depth of the upper water layer. These results demonstrate that phytoplankton blooms can develop in the absence of vertical water-column stratification.
\end{abstract}

A common paradigm in aquatic ecology and oceanography is that phytoplankton blooms can develop only if the upper mixed-water layer is shallower than some critical depth (e.g., Sverdrup 1953; Smetacek and Passow 1990; Nelson and Smith 1991; Platt et al. 1991; Kirk 1994; Mann and Lazier 1996; Obata et al. 1996; Falkowski and Raven 1997). The idea is that once the upper water column is sufficiently shallow, the light conditions will be favorable for photosynthesis, so that depth-integrated photosynthesis exceeds the depth-integrated losses of the phytoplankton. Hence, the phytoplankton population increases.

This critical depth concept was recently challenged by Townsend et al. (1992) and Eilertsen (1993). Townsend et al. (1992) reported that the phytoplankton spring bloom in the Gulf of Maine preceded the onset of vertical water-column stratification. Similarly, Eilertsen (1993) observed that in several Norwegian fjords with water depths of $>200 \mathrm{~m}$, phytoplankton blooms developed in the absence of vertical water-column stratification throughout. These observations were criticized by Platt et al. (1994) and Mann and Lazier (1996), basically for two reasons. First, it was not clear whether the observations of Townsend et al. and Eilertsen indeed violated the critical depth concept. It could be that the mixed-layer depth was misjudged, that the phytoplankton losses were unusually low, or that the production rates were unexpectedly high. Second, if the observations of Townsend et al. and Eilertsen did violate the critical depth concept, a proper theory to explain their observations was lacking.

Here, we develop a new theory that provides a theoretical underpinning for the observations of Townsend et al. (1992) and Eilertsen (1993), as well as for many other observations. The mathematical models that underlie the critical depth concept assume that the phytoplankton population is homogeneously distributed over depth (Sverdrup 1953; Platt et al. 1991). We relax this crucial assumption, as it may not hold in many aquatic environments. Our strategy will be to let the dynamics of phytoplankton growth and turbulent mixing determine the phytoplankton distribution over depth. The analysis reveals that there are two different mechanisms for the development of phytoplankton blooms. A bloom can develop if the upper mixed-water layer is shallower than some critical depth or if turbulent mixing rates are less than some critical turbulence.

The model-We consider a growth-diffusion model (Okubo 1980) in which the population dynamics of phytoplankton are governed by light-limited growth, local phytoplankton losses, and local transport of the phytoplankton by turbulent diffusion. Following Sverdrup (1953), the model is kept as simple as possible. Thus, the model neglects many additional complexities like nutrient limitation, photoinhibition, buoyancy regulation, and sinking, in order to focus on the most fundamental aspects of phytoplankton bloom development. Our diffusion equation forms the core of various more complicated models used in oceanography and ecosystems research (Cloern 1991; Slagstad and Støle-Hansen 1991; Koseff et al. 1993; Donaghay and Osborn 1997; Lucas et al. 1998) and has attracted considerable mathematical treatment (Shigesada and Okubo 1981; Ishii and Takagi 1982; Totaro 1989), but its features have never been fully analyzed.

Phytoplankton dynamics: Let $s$ denote the depth within the water column, where $s$ runs from zero (top) to $z$ (bottom). And let $\omega(s, t)$ denote the phytoplankton population density (cells per unit volume) at depth $s$ and time $t$. The changes in population density can be described by a partial differential equation.

$$
\frac{\partial \omega}{\partial t}(s, t)=[p(I(s, t))-\ell] \omega(s, t)+D \frac{\partial^{2} \omega}{\partial s^{2}}(s, t) .
$$

Here, $p(I(s, t))$ is the specific production rate as an increasing function of light intensity $I(s, t)$, with $p(0)=0 ; \ell$ is the specific loss rate, which incorporates all sources of phytoplankton loss; and $D$ is the turbulent diffusion coefficient (also known as "vertical eddy diffusivity"). For notational convenience, we introduce the net production rate, $g(I)=$ $p(I)-\ell$.

We assume that the water column is closed, with no phytoplankton cells entering or leaving the column at the top or the bottom. This gives the following boundary conditions:

$$
\frac{\partial \omega}{\partial s}(0, t)=\frac{\partial \omega}{\partial s}(z, t)=0 .
$$

It is useful to keep track not only of the population density 
distribution (cells per unit volume) but also of the total population size per unit surface area, $W$, which is given by

$$
W(t)=\int_{0}^{z} \omega(s, t) d s .
$$

Combining Eqs. 1-3, the population size per unit surface area changes with time according to

$$
\frac{d W}{d t}=\int_{0}^{z} \frac{\partial \omega}{\partial t}(s, t) d s=\int_{0}^{z} g(I(s, t)) \omega(s, t) d s .
$$

Light gradient: The light gradient follows Lambert-Beer's law, which states that the amount of light absorbed at depth $s$ is proportional to the light intensity at depth $s$ :

$$
\frac{\partial I}{\partial s}(s, t)=-\kappa(s, t) I(s, t) .
$$

The constant of proportionality, $\kappa(s, t)$, is based on all components that absorb light, including the water itself and the phytoplankton:

$$
\kappa(s, t)=K_{b g}+k \omega(s, t),
$$

where $K_{b g}$ is the total background turbidity due to nonphytoplankton components, and $k$ is the specific light attenuation coefficient of the phytoplankton. Combining Eqs. 5 and 6 and integrating over depth gives the following light intensity at depth $s$ and time $t$ :

$$
I(s, t)=I_{\text {in }} \exp \left[-\left(k \int_{0}^{s} \omega(\sigma, t) d \sigma+K_{b g} s\right)\right]
$$

where $I_{\text {in }}$ is the incident light intensity, and $\sigma$ is an integration variable. Note that our formulation explicitly includes light absorption by phytoplankton. Thus, the light gradient is dynamic - it changes with a change in the phytoplankton population densities.

Vertical distribution patterns-Classical critical depth theory is based on the assumption that the phytoplankton are homogeneously distributed over depth (Sverdrup 1953; Platt et al. 1991). In contrast, the combination of growth and turbulent diffusion in a light gradient generates an inhomogeneous vertical distribution of the phytoplankton population. More precisely, the stationary distribution of population density over depth can be obtained by equating the right-hand side of Eq. 1 with zero. This yields

$$
\frac{d^{2} \omega^{*}}{d s^{2}}=-\frac{1}{D} g(I(s)) \omega^{*}(s),
$$

where the superscript $*$ indicates that the population density distribution is evaluated at steady state. Integrating both sides with respect to depth, and using Eq. 2 to find that the constant of integration equals zero, we get the slope of the depth profile:

$$
\frac{d \omega^{*}}{d s}=-\frac{1}{D} \int_{0}^{s} g(I(\sigma)) \omega^{*}(\sigma) d \sigma
$$

Note that the integration boundaries in Eq. 9 range from zero

\section{Population density $\left(10^{4}\right.$ cells $\left.\mathrm{ml}^{-1}\right)$}

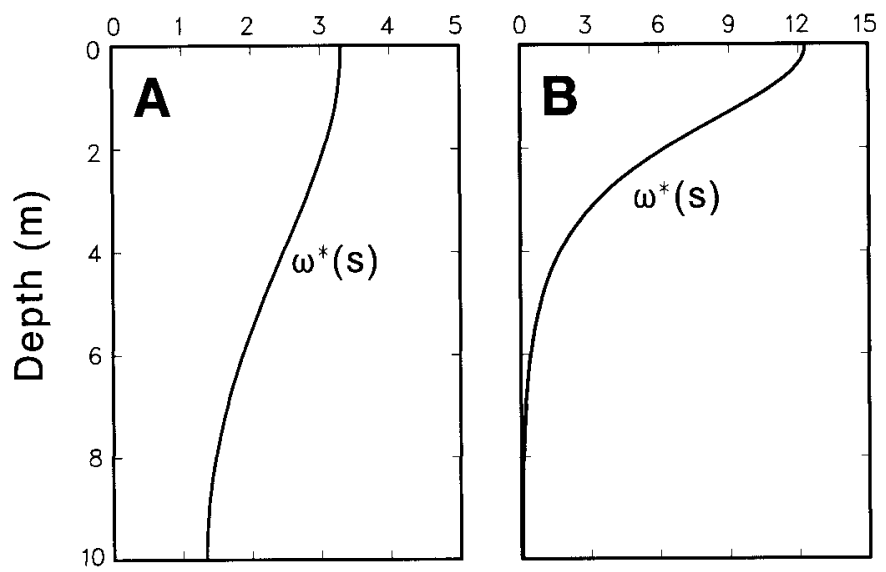

Fig. 1. Depth profile of the steady-state phytoplankton population density, $\omega^{*}(s)$, when the phytoplankton dynamics are governed by light availability and turbulent diffusion. The population density decreases with depth, with a point of inflection at the compensation point. In (A), turbulent mixing is relatively intense $\left(D=1 \mathrm{~cm}^{2} \mathrm{~s}^{-1}\right.$; $z=10 \mathrm{~m})$; in (B), turbulent mixing is relatively weak $(D=0.1$ $\left.\mathrm{cm}^{2} \mathrm{~s}^{-1} ; z=10 \mathrm{~m}\right)$. Other parameter values are given in the legend of Fig. 2.

to $s$, whereas they range from zero to $z$ in Eq. 4 . Hence, using $d W / d t=0$ at steady state and the monotonicity of $g(I)$, Eq. 9 shows that the stationary population density should decrease monotonically with depth. That is, if $\omega^{*}(s)>0$,

$$
\frac{d \omega^{*}}{d s}<0 \text { for } 0<s<z
$$

Furthermore, upon defining the compensation point $I_{c}$ as $g\left(I_{c}\right)=0$, it follows from Eq. 8 that, if $\omega^{*}(s)>0$,

$\frac{d^{2} \omega^{*}}{d s^{2}}<0 \quad$ for $I>I_{c}, \quad$ and $\quad \frac{d^{2} \omega^{*}}{d s^{2}}>0 \quad$ for $I<I_{c}$.

We now have sufficient information to sketch the qualitative shape of the stationary distribution of the phytoplankton population over depth. According to the above derivations, there are two possibilities: (1) there is no phytoplankton population at all, i.e., the stationary population density distribution remains zero throughout the water column; or (2) the stationary population density distribution is positive for at least some parts of the water column (Fig. 1). In the latter case, the population density should decrease with increasing depth (Eq. 10), with zero slopes at the top and bottom of the water column (Eq. 2) and a point of inflection at the compensation point (Eq. 11). The steepness of the profile depends on the magnitude of the turbulent diffusion coefficient relative to the depth-integrated phytoplankton growth rate (Eq. 9). Depending on the precise parameter settings, the stationary population density distribution either may remain positive throughout the water column (as in Fig. 1A) or it may become zero at some depth above the bottom of the water column (as in Fig. 1B).

A rigorous stability analysis performed by Ishii and Takagi (1982) shows that, for the class of nonlinear diffusion 
equations considered here, the stationary solution is unique and globally stable. In other words, the model predicts that, independent of the initial conditions, the vertical population density distribution will always tend toward the stationary population density distribution.

It is obvious that other vertical distributions may develop if other processes (such as photoinhibition, sinking, algal motility, or nutrient limitation) are involved. However, in the real world, the vertical distribution patterns of Fig. 1 are quite frequently observed in dense phytoplankton blooms (see fig. 29 in Reynolds 1984 for examples from a freshwater lake, Eilertsen 1993 for an example from a Norwegian fjord, and Morel 1997 for an example from the tropical Atlantic Ocean offshore Mauritania). The resemblance between these depth profiles and the depth profiles of Fig. 1 is striking.

Conditions for phytoplankton bloom development-We used numerical simulations of Eqs. 1-7 to explore under what conditions phytoplankton blooms occur. For this purpose, we defined the conditions for phytoplankton bloom development simply as those conditions that are able to support a positive steady-state population (i.e., $\mathrm{W}^{*}>0$ ). Because the stationary population density distribution is a global attractor, this definition is equivalent to Sverdrup's (1953) original definition of net population growth at low population size (i.e., $d W / d t>0$ for $W$ close to zero).

The simulations revealed that phytoplankton blooms can develop (1) if the depth of the mixed layer is less than a critical depth, and/or (2) if the turbulent mixing is less than a critical turbulence (Fig. 2). Most strikingly, the area of no blooms in the upper right of Fig. 2 is bound by nearly horizontal and vertical lines. That is, the critical depth is independent of the turbulence level, and the critical turbulence is independent of water-column depth. Hence, there are two distinct and independent mechanisms for the development of a phytoplankton bloom. Either mixing is intense, and a shallowing of the mixed-layer depth is necessary to expose the phytoplankton to the favorable light conditions, or mixing is weak, and relaxation of turbulent mixing allows the phytoplankton growth rates to overcome the vertical mixing rates imposed by turbulent diffusion. The latter mechanism also maintains the phytoplankton in the upper part of the water column (see Fig. 1B), so that its growth dynamics become independent of processes further downward.

What will be the population size of a bloom? To address this question, we used the model to calculate the steady-state population size per unit surface area along four different transects indicated in Fig. 2. This revealed that population size per unit surface area decreases linearly with water-column depth in well-mixed environments, whereas it is independent of water-column depth in environments with low mixing rates (Fig. 3A). Furthermore, the model predicts that population size per unit surface area decreases strongly with increasing turbulent mixing in deep water columns but is independent of turbulent mixing in very shallow water columns (Fig. 3B). The solid line in Fig. 3A is consistent with the results of recent laboratory experiments with well-mixed continuous cultures (Huisman 1999). The dashed line in Fig. 3B is consistent with recent field data from phytoplankton blooms in Lake Kinneret, Israel (Berman and Shteinman

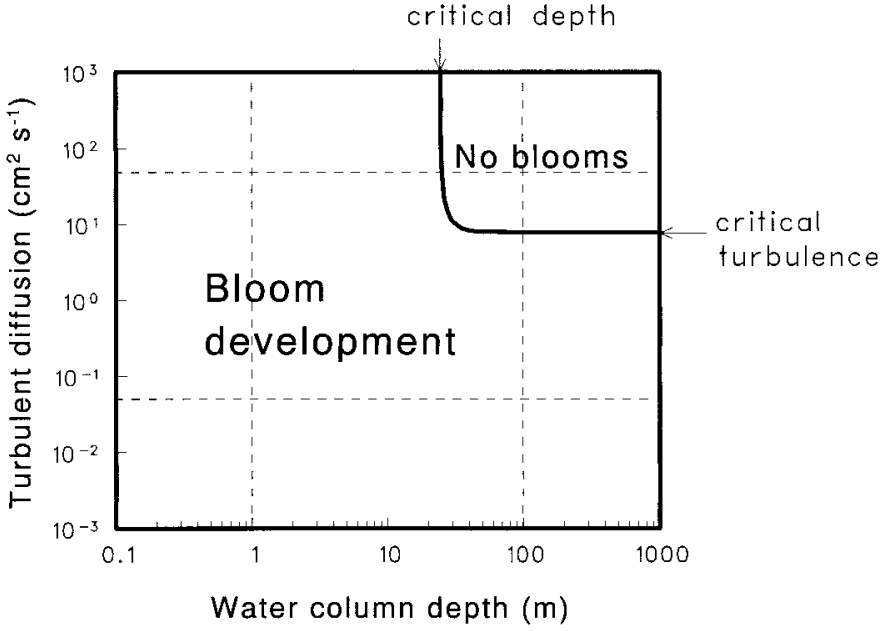

Fig. 2. The combinations of water-column depth and turbulent diffusion coefficient that allow a phytoplankton bloom $\left(W^{*}>0\right)$ and the combinations that prevent a phytoplankton bloom $\left(W^{*}=\right.$ $0)$. The two regions are separated by a solid line that runs practically parallel with the coordinate axes, thus indicating the existence of a critical depth and a critical turbulence. The graph is based on extensive simulations of Eqs. 1-7, using the parameter set $I_{\text {in }}=350$ $\mu \mathrm{mol}$ photons $\mathrm{m}^{-2} \mathrm{~s}^{-1} ; K_{b g}=0.2 \mathrm{~m}^{-1} ; g(I)=0.04 \cdot I /(30+I)-$ $0.02 \mathrm{~h}^{-1} ; k=0.15 \times 10^{-6} \mathrm{~cm}^{2}$ cell ${ }^{-1}$. To test the robustness of our findings, many other parameter sets were also examined. They all yielded the same pattern: phytoplankton blooms are possible if water-column depth is below a certain critical depth or if turbulent diffusion is below a certain critical turbulence. Note the log scales: the graph spans the entire spectrum from extremely shallow and quiescent waters to extremely deep and turbulent waters. The thin dashed lines indicate the locations of the four transects shown in Fig. 3.

1998). The various patterns can be explained by the fact that phytoplankton have to compete for light with the background turbidity (Huisman and Weissing 1994, 1995; Kirk 1994; Carpenter et al. 1998; Huisman et al. 1999b). Dense phytoplankton blooms can develop only if shading by background turbidity is low, i.e., in deep waters where low turbulent mixing rates allow vertical escape from shading (dashed line in Fig. 3A; left part of dashed line in Fig. 3B) or in shallow water layers (left part of solid line in Fig. 3A; solid line in Fig. 3B). This again illustrates that both watercolumn depth and turbulent mixing rates have a profound impact on bloom development.

Properties of critical depth and critical turbulence-Critical depth and critical turbulence are functions of the phytoplankton characteristics and the environment.

Critical depth: An exact analytical expression for the critical depth can be derived from the concept of a critical light intensity (Huisman and Weissing 1994; also see Weissing and Huisman 1994; Huisman 1999; Huisman et al. 1999a,b). That is, if $Z_{c r}$ denotes the critical depth and $I_{\text {out }}^{*}$ denotes the critical light intensity, then the critical depth is given by (Huisman 1999) 

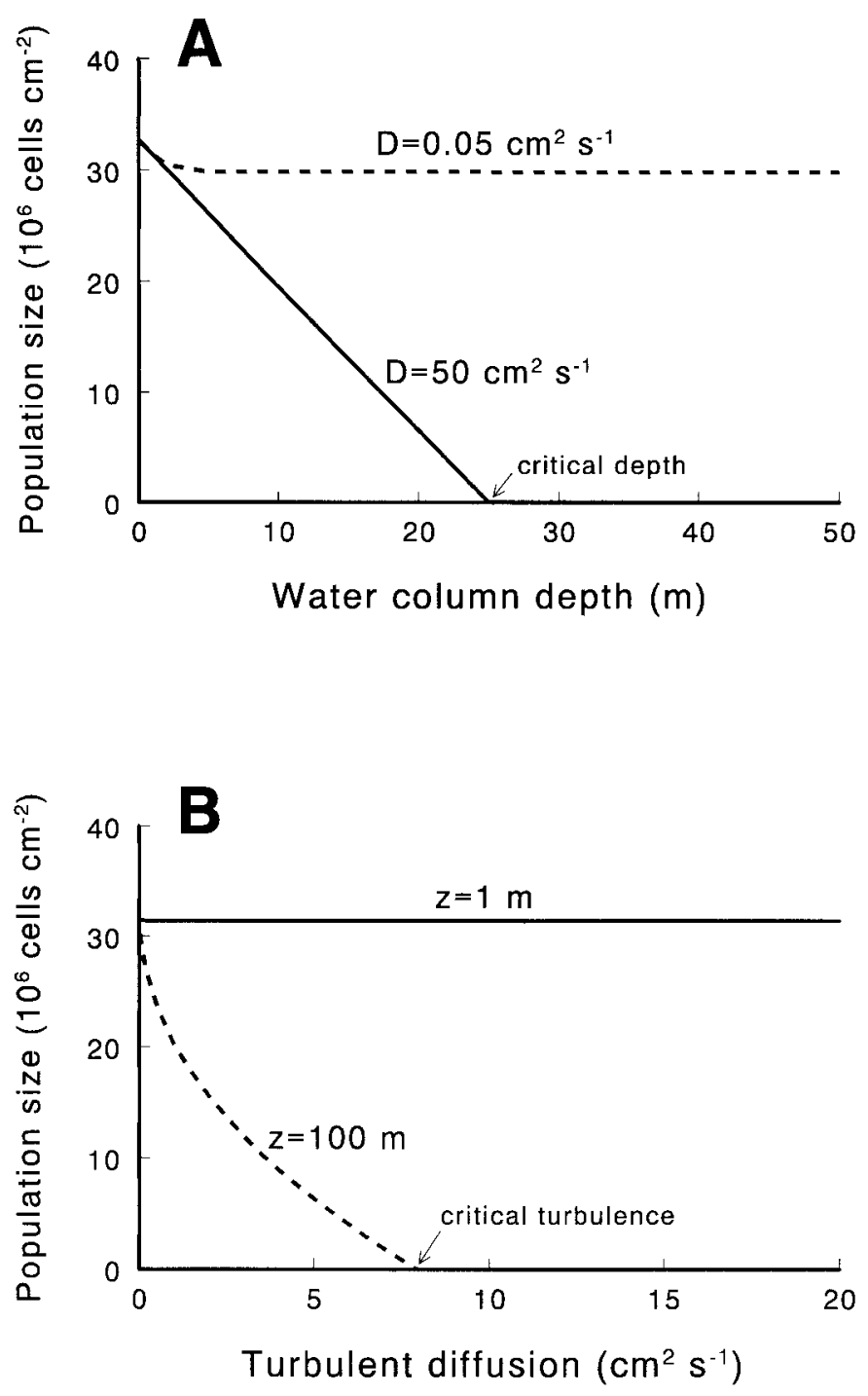

Fig. 3. Phytoplankton bloom size. (A) Steady-state phytoplankton population size per unit surface area in relation to water-column depth for two different turbulence levels. (B) Steady-state phytoplankton population size per unit surface area in relation to the turbulent diffusion coefficient for two different water-column depths. The locations of the four transects are indicated in Fig. 2, and the parameter values are given in the legend of Fig. 2.

$$
Z_{c r}=\frac{\ln \left(I_{\text {in }}\right)-\ln \left(I_{\text {out }}^{*}\right)}{K_{b g}} .
$$

Here, the critical light intensity is defined as that value of the light intensity at the bottom of a well-mixed water column for which the phytoplankton population integrated over the entire water column remains stationary. Analytically, it can be shown that the critical light intensity is given by the implicit solution of (Huisman and Weissing 1994; Weissing and Huisman 1994)

$$
\int_{I_{\text {out }}^{*}}^{I_{\text {in }}} \frac{g(I)}{k I} d I=0 .
$$

Weissing and Huisman (1994) give formal proof that this expression for the critical light intensity holds independent of the precise mathematical equation used for the $g(I)$ function. The critical light intensity is species specific. It follows, via Eq. 12, that the critical depth should be species specific as well. Blooms dominated by species with different light requirements should have different critical depths. These theoretical predictions are consistent with results of extensive laboratory experiments with monocultures and mixtures of phytoplankton species (Huisman 1999; Huisman et al. 1999a).

The critical depths predicted by Eqs. 12 and 13 correspond exactly with the critical depths observed in our numerical simulations. It follows that the critical depth is inversely proportional to background turbidity (Eq. 12). Furthermore, via Eq. 13, the critical depth increases with incident light intensity, increases with phytoplankton production rates, and decreases with phytoplankton loss rates. Our formulation of the critical depth generalizes earlier formulations by, among others, Sverdrup (1953), Platt et al. (1991), and Kirk (1994).

Critical turbulence: We have not been able to derive an analytical expression for the critical turbulence. Some insight, however, can be gained using dimensional analysis. Let us rescale Eqs. 1-7 by introducing the new variables $\hat{s}$ $=c s$ and $\hat{\omega}=\omega / c$, where $c$ is some arbitrary constant. This leads to an equivalent system with new parameters:

$$
\hat{K}_{b g}=K_{b g} / c, \quad \hat{z}=c z, \quad \hat{D}=c^{2} D .
$$

These new parameters summarize the scaling rules of the system. Thus, the dynamics of the system remain unaltered if changing the background turbidity by a factor $1 / c$ is compensated by changing water-column depth by a factor $c$ and the turbulent diffusion coefficient by a factor $c^{2}$. Remember from Fig. 2 that the critical depth is independent of the turbulent diffusion coefficient. Hence, it follows from the scaling rules that the critical depth is inversely proportional to the background turbidity (in line with Eq. 12). Remember also from Fig. 2 that the critical turbulence is independent of water-column depth. Hence, it follows from the scaling rules that the critical turbulence is inversely proportional to the square of background turbidity:

$$
D_{c r} \propto \frac{1}{\left(K_{b g}\right)^{2}} .
$$

This is confirmed by the simulations (Fig. 4). Furthermore, extensive simulations indicate that the critical turbulence is species specific, increases with incident light intensity, increases with phytoplankton production rates, and decreases with phytoplankton loss rates.

Application: Note from Eqs. 12 and 15 that the critical depth and the critical turbulence approach infinity when background turbidity approaches zero. In other words, without background turbidity, there would be neither a critical depth nor a critical turbulence to delimit phytoplankton growth. Note also that the critical turbulence increases faster with decreasing background turbidity than the critical depth (compare the denominators of Eqs. 12 and 15). This suggests that, all other parameters held constant, the critical-turbu- 


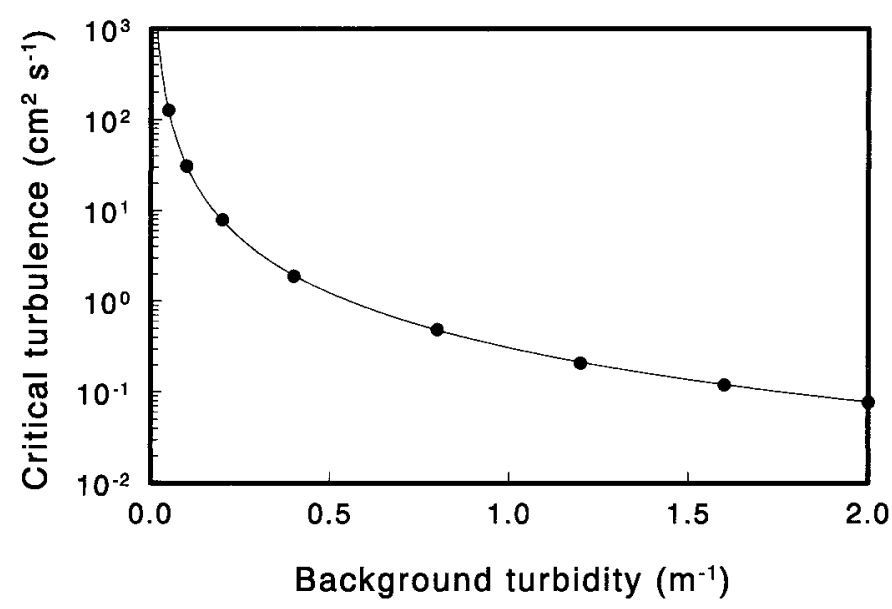

Fig. 4. The critical turbulence decreases with the square of background turbidity. Closed circles show the critical-turbulence levels estimated from the simulations, using the parameter values given in the legend of Fig. 2. The line that goes through these points is the curve $D_{c r}=0.31 /\left(K_{b g}\right)^{2}$.

lence mechanism will be relatively more important in waters with a low background turbidity than in waters with a high background turbidity. Therefore, we conjecture that the critical-turbulence mechanism should be relatively more important in the open ocean or in clear lakes than in the turbid coastal zone or in turbid lakes.

To illustrate this point further, consider an example with real-world parameter estimates. The phytoplankton parameter values that we chose for the construction of Figs. 1-4 are typical for the "average" freshwater phytoplankton species in the culture collection of the Laboratory for Microbiology, University of Amsterdam, The Netherlands (De Nobel et al. 1998; Huisman 1999; Huisman et al. 1999a). Background turbidity in natural waters ranges, according to Kirk (1994:291), from $K_{b g}=0.08 \mathrm{~m}^{-1}$ in clear oceanic waters to $K_{b g}=2.0 \mathrm{~m}^{-1}$ in highly turbid lakes. Hence, using Fig. 4, the critical turbulence for our "average" phytoplankton species ranges from $D_{c r}=48 \mathrm{~cm}^{2} \mathrm{~s}^{-1}$ in clear oceanic waters to $D_{c r}=0.08 \mathrm{~cm}^{2} \mathrm{~s}^{-1}$ in turbid lakes. In other words, blooms develop via the critical-turbulence mechanism if the vertical turbulent diffusion coefficient is $<48 \mathrm{~cm}^{2} \mathrm{~s}^{-1}$ in the clearest oceanic waters and if it is $<0.08 \mathrm{~cm}^{2} \mathrm{~s}^{-1}$ in the most turbid lakes. For comparison, vertical turbulent diffusion coefficients observed in lakes and oceans typically vary from $D=0.1 \mathrm{~cm}^{2} \mathrm{~s}^{-1}$ during quiescent periods to $D=100 \mathrm{~cm}^{2}$ $\mathrm{s}^{-1}$ during episodes of intense mixing (Officer 1976; Denman and Gargett 1983; MacIntyre 1993). This comparison illustrates two points. First, the critical turbulences that we estimated are within the realistic range, which suggests that the critical-turbulence mechanism may apply to real-world situations. Second, these numerical estimates show, once again, that the critical-turbulence mechanism for bloom development is much more likely to work in clear waters than in turbid waters.

Sinking and buoyancy-Thus far, the analysis has assumed that phytoplankton transport is governed by turbulent diffusion. Other vertical transport processes relevant for phy-

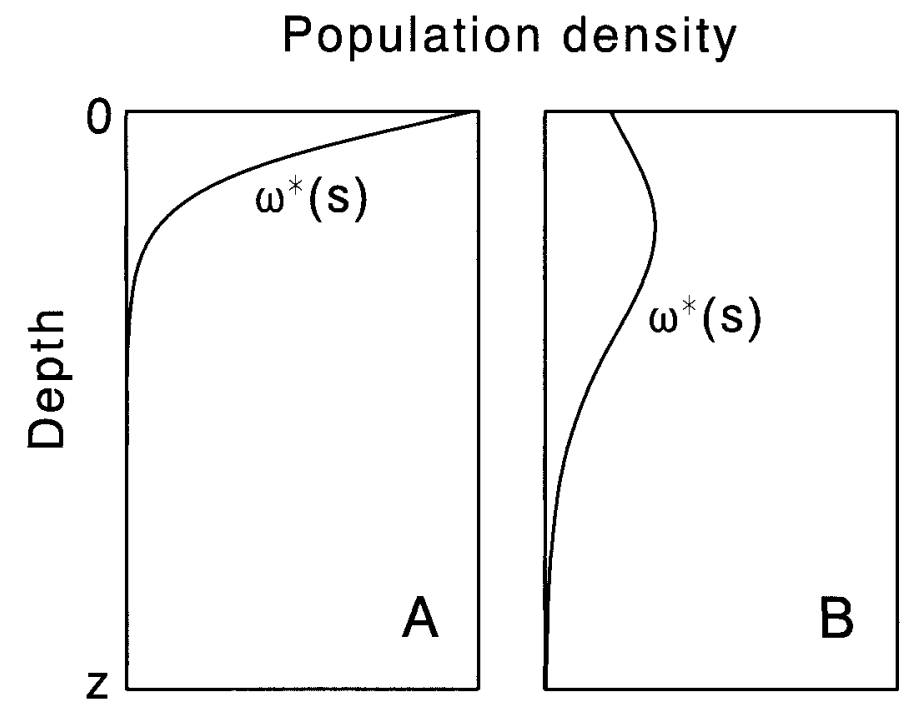

Fig. 5. Depth profiles of the steady-state phytoplankton population density, $\omega^{*}(s)$, sketched for (A) a buoyant phytoplankton species, and (B) a sinking phytoplankton species.

toplankton include sinking (e.g., diatoms) and buoyancy (e.g., several cyanobacterial species). How do sinking and buoyancy of phytoplankton affect the derivations?

Sinking and buoyancy are incorporated in the theoretical framework by adding an advection term to the model. Equation 1 is replaced by

$$
\frac{\partial \omega}{\partial t}(s, t)=g(I(s, t)) \omega(s, t)+v \frac{\partial \omega}{\partial s}(s, t)+D \frac{\partial^{2} \omega}{\partial s^{2}}(s, t)
$$

where $v$ is the vertical velocity of the phytoplankton, which is positive for buoyant phytoplankton and negative for sinking phytoplankton. In addition, Eq. 2 is replaced by new zero-flux boundary conditions:

$$
v \omega(s, t)+D \frac{\partial \omega}{\partial s}(s, t)=0 \quad \text { at } s=0 \quad \text { and } \quad s=z .
$$

All other aspects of the model formulation (Eqs. 3-7) remain the same.

Analogous to the earlier derivations, the stationary population density distribution can be derived by equating the right-hand side of Eq. 16 with zero and subsequent integration over depth. This gives the slope of the depth profile (compare with Eq. 9):

$$
\frac{\partial \omega^{*}}{\partial s}=-\frac{1}{D} \int_{0}^{s} g(I(\sigma)) \omega^{*}(\sigma) d \sigma-\frac{v}{D} \omega^{*}(s) .
$$

Furthermore, according to Eq. 17, the slopes of the stationary population density distribution at the top and bottom of the water column are given by

$$
\frac{\partial \omega^{*}}{\partial s}=-\frac{v}{D} \omega^{*}(s) \quad \text { at } s=0 \quad \text { and } \quad s=z .
$$

This provides the information to sketch the stationary profile of phytoplankton population density over depth (Fig. 5). It follows from Eq. 4, using $d W / d t=0$, that the first term in Eq. 18 is negative. Hence, the sign and magnitude of the 
second term in Eq. 18 determine whether the depth profile has a positive or negative slope. For buoyant phytoplankton $(v>0)$, Eqs. 18 and 19 predict that the slope is negative. That is, population density is a strictly decreasing function of depth (Fig. 5A). All else being equal, buoyant phytoplankton aggregate further in top of the water column than phytoplankton of neutral weight (compare Eqs. 9 and 18). For sinking phytoplankton $(v<0)$, Eqs. 18 and 19 allow population density distributions of various shapes. For example, sinking phytoplankton may simply be lost from the water column and accumulate at the bottom, particularly if sinking rates are high and turbulent diffusion is low (see Visser et al. 1996b; Lucas et al. 1998). Alternatively, if phytoplankton with a high sinking velocity are able to maintain a population in the upper water column (i.e., $\omega^{*}(0)>0$ ), then Eq. 19 predicts that the slope of the depth profile in top of the water column will always be positive. Figure 5B gives an example. Hence, all else being equal, sinking phytoplankton aggregate less in top of the water column than phytoplankton of neutral weight (again, compare Eqs. 9 and 18).

The critical turbulence is based on the notion that phytoplankton maintain a population in the upper water column if growth rates in the upper water column exceed rates of vertical transport downward. Buoyant phytoplankton aggregate more and sinking phytoplankton aggregate less in the upper water column than phytoplankton of neutral weight (Fig. 5A,B). Hence, in conclusion, the critical-turbulence mechanism for bloom development will be relatively less important for sinking phytoplankton than for phytoplankton of more or less neutral weight. Conversely, the critical-turbulence mechanism will be relatively more important for buoyant phytoplankton species.

Conclusions-We have shown that phytoplankton blooms can be induced by two fundamentally different mechanisms. The formation of a relatively shallow mixed-water layer by means of vertical water-column stratification is prerequisite for phytoplankton bloom development in deep and/or turbid waters with high turbulent mixing rates. This mechanism corresponds to Sverdrup's (1953) critical depth concept. Alternatively, relaxation of turbulent mixing in waters of low background turbidity allows bloom development irrespective of the thickness of the upper water column. In the latter mechanism, the phytoplankton bloom maintains its position in the upper part of the water column because the phytoplankton growth rates in the upper part of the water column exceed the vertical mixing rates. The two different mechanisms are effectively independent of one another (Fig. 2).

Our findings are consistent with theory and experiments for well-mixed environments (Sverdrup 1953; Platt et al. 1991; Huisman and Weissing 1994; Huisman 1999). At the same time, our findings support the observations of Townsend et al. (1992) and Eilertsen (1993) that phytoplankton blooms can also develop in the absence of vertical watercolumn stratification. Consistent with the theory developed here, these observations were made in clear waters during periods with weak vertical wind mixing. Similarly, long-term data show that phytoplankton blooms in estuaries often form during neap tide, when turbulent mixing is at its weakest (Cloern 1991). Other recent field studies that illustrate the impact of turbulent mixing rates on phytoplankton bloom development include those of Ragueneau et al. (1996), Visser et al. (1996a), Yin et al. (1996), and Berman and Shteinman (1998).

It is conceivable that the two different mechanisms for bloom development (critical depth and critical turbulence) lead to a difference in species composition as well. A multispecies extension of the model revealed that the two mechanisms indeed favor different phytoplankton strategies (Huisman et al. 1999b). The multispecies model predicts that well-mixed waters favor the species with lowest critical light intensity or, equivalently, the species with deepest critical depth (Huisman and Weissing 1994). Competition experiments support this prediction (Huisman et al. 1999a). Incomplete mixing, in contrast, favors species that are able to obtain the best vertical positions within a water column. Accordingly, the species composition in well-mixed waters should generally differ from the species composition in waters of low turbulence (Huisman et al. 1999b).

Interestingly, low vertical mixing rates favor not only phytoplankton bloom development but also the development of a temperature gradient. In this way, the development of temperature gradients and phytoplankton blooms may coincide without any causal relationship. This coincidence probably explains why, independent of the mechanism of bloom development involved, the timing of bloom development often matches the timing of water-column stratification and thus why the critical-turbulence mechanism outlined in this paper has not been discovered earlier.

Jef Huisman ${ }^{1}$

Biological Sciences

Stanford University

Stanford, California 94305-5020

Paul van Oostveen ${ }^{2}$ Franz J. Weissing

Department of Genetics

University of Groningen

P.O. Box 14

9750 AA Haren, The Netherlands

\section{References}

Berman, T., AND B. Shteinman. 1998. Phytoplankton development and turbulent mixing in Lake Kinneret (1992-1996). J. Plankton Res. 20: 709-726.

\footnotetext{
${ }^{1}$ Present address: Laboratory for Microbiology, University of Amsterdam, Nieuwe Achtergracht 127, 1018 WS Amsterdam, The Netherlands.

${ }^{2}$ Present address: Ministry of Foreign Affairs, P.O. Box 20061, 2500 EB The Hague, The Netherlands.

\section{Acknowledgments}

We thank Sean Connolly, Lisa Lucas, Stephen Monismith, Joan Roughgarden, and the anonymous referees for their helpful comments. The investigations of J.H. were supported by a TALENT grant from the Netherlands Organization for Scientific Research (NWO) and by a grant from the Earth and Life Sciences Foundation (ALW), which is subsidized by NWO. This is publication 2539 of the Center for Estuarine and Marine Ecology, Yerseke.
} 
Carpenter, S. R., J. J. Cole, J. F. Kitchell, and M. L. Pace. 1998. Impact of dissolved organic carbon, phosphorus, and grazing on phytoplankton biomass and production in experimental lakes. Limnol. Oceanogr. 43: 73-80.

Cloern, J. E. 1991. Tidal stirring and phytoplankton bloom dynamics in an estuary. J. Mar. Res. 49: 203-221.

Denman, K. L., AND A. E. Gargett. 1983. Time and space scales of vertical mixing and advection of phytoplankton in the upper ocean. Limnol. Oceanogr. 28: 801-815.

De Nobel, W. T., H.C.P. Matthiss, E. Von Elert, and L. R. Mur. 1998. Comparison of the light-limited growth of the nitrogenfixing cyanobacteria Anabaena and Aphanizomenon. New Phytol. 138: 579-587.

Donaghay, P. L., AND T. R. Osborn. 1997. Toward a theory of biological-physical control of harmful algal bloom dynamics and impacts. Limnol. Oceanogr. 42: 1283-1296.

EILERTSEN, H. C. 1993. Spring blooms and stratification. Nature 363: 24.

Falkowski, P. G., AND J. A. Raven. 1997. Aquatic photosynthesis. Blackwell.

Huisman, J. 1999. Population dynamics of light-limited phytoplankton: Microcosm experiments. Ecology 80: 202-210.

, R. R. Jonker, C. ZonNeveld, AND F. J. WeIssing. 1999a. Competition for light between phytoplankton species: Experimental tests of mechanistic theory. Ecology 80: 211-222.

— , P. van Oostveen, and F. J. Weissing. 1999b. Species dynamics in phytoplankton blooms: Incomplete mixing and competition for light. Am. Nat. 154: 46-68.

—, AND F. J. WeIssing. 1994. Light-limited growth and competition for light in well-mixed aquatic environments: An elementary model. Ecology 75: 507-520.

— AND 1 1995. Competition for nutrients and light in a mixed water column: A theoretical analysis. Am. Nat. 146: 536-564.

IsHII, H., AND I. TAKAGI. 1982. Global stability of stationary solutions to a nonlinear diffusion equation in phytoplankton dynamics. J. Math. Biol. 16: 1-24.

KIRK, J.T.O. 1994. Light and photosynthesis in aquatic ecosystems, 2nd ed. Cambridge Univ. Press.

Koseff, J. R., J. K. Holen, S. G. Monismith, and J. E. Cloern. 1993. Coupled effects of vertical mixing and benthic grazing on phytoplankton populations in shallow, turbid estuaries. J. Mar. Res. 51: 843-868.

Lucas, L. V., J. E. Cloern, J. R. Koseff, S. G. Monismith, and J. K. Thompson. 1998. Does the Sverdrup critical depth model explain bloom dynamics in estuaries? J. Mar. Res. 56: 375415.

MACINTYRE, S. 1993. Vertical mixing in a shallow, eutrophic lake: Possible consequences for the light climate of phytoplankton. Limnol. Oceanogr. 38: 798-817.

MANN, K. H., AND J.R.N. LAZIER. 1996. Dynamics of marine ecosystems: Biological-physical interactions in the oceans, 2nd ed. Blackwell.

Morel, A. 1997. Consequences of a Synechococcus bloom upon the optical properties of oceanic (case 1) waters. Limnol. Oceanogr. 42: 1746-1754.

Nelson, D. M., AND W. O. SMith, JR. 1991. Sverdrup revisited: Critical depths, maximum chlorophyll levels, and the control of Southern Ocean productivity by the irradiance-mixing regime. Limnol. Oceanogr. 36: 1650-1661.

OBATA, A., J. IshIZAKA, AND M. ENDOH. 1996. Global verification of critical depth theory for phytoplankton bloom with climatological in situ temperature and satellite ocean color data. J. Geophys. Res. 101: 20657-20667.

OFFICER, C. B. 1976. Physical oceanography of estuaries (and associated coastal waters). Wiley.

OkuBo, A. 1980. Diffusion and ecological problems: Mathematical models. Springer.

Platt, T., D. F. Bird, and S. Sathyendranath. 1991. Critical depth and marine primary production. Proc. R. Soc. Lond. B 246: 205-217.

, J. D. Woods, S. SAThyendranath, AND W. BARKMANN. 1994. Net primary production and stratification in the ocean, p. 247-254. In D. M. Johannesen, R. D. Muench, and D. E. Overland [eds.], The polar oceans and their role in shaping the global environment. Geophysical Monogr. 85. American Geophysical Union.

Ragueneau, O., B. Quéguiner, And P. Trégeur. 1996. Contrast in biological responses to tidally-induced vertical mixing for two macrotidal ecosystems of western Europe. Estuarine Coastal Shelf Sci. 42: 645-665.

REYNOLDS, C. S. 1984. The ecology of freshwater phytoplankton. Cambridge Univ. Press.

ShigesadA, N., AND A. OKubo. 1981. Analysis of the self-shading effect on algal vertical distribution in natural waters. J. Math. Biol. 12: 311-326.

Slagstad, D., And K. Støle-Hansen. 1991. Dynamics of plankton growth in the Barents Sea: Model studies. Polar Res. 10: 173186.

SmetaceK, V., AND U. PAssow. 1990. Spring bloom initiation and Sverdrup's critical-depth model. Limnol. Oceanogr. 35: 228234.

SVERDRUP, H. U. 1953. On conditions for the vernal blooming of phytoplankton. J. Cons. Perm. Int. Explor. Mer 18: 287-295.

TotARO, S. 1989. Mutual shading effect on algal distribution: A nonlinear problem. Nonlinear Anal. Theor. Methods Appl. 13: 969-986.

Townsend, D. W., M. D. Keller, M. E. Sieracki, and S. G. ACKLESON. 1992. Spring phytoplankton blooms in the absence of vertical water column stratification. Nature 360: 59-62.

Visser, P. M., B. W. Ibelings, B. VAn DeR Veer, J. Koedood, AND L. R. MUR. 1996a. Artificial mixing prevents nuisance blooms of the cyanobacterium Microcystis in Lake Nieuwe Meer, The Netherlands. Freshwater Biol. 36: 435-450.

, L. Massaut, J. Huisman, and L. R. Mur. 1996b. Sedimentation losses of Scenedesmus in relation to mixing depth. Arch. Hydrobiol. 136: 289-308.

Weissing, F. J., AND J. HuISMAn. 1994. Growth and competition in a light gradient. J. Theor. Biol. 168: 323-336.

Yin, K., P. J. Harrison, R. H. Goldblatt, and R. J. Beamish. 1996. Spring bloom in the central Strait of Georgia: Interactions of river discharge, winds and grazing. Mar. Ecol. Prog. Ser. 138: 255-263.

Received: 6 July 1998 Accepted: 14 May 1999 Amended: 26 May 1999 